Article

\title{
The Stability Analysis of a Multi-Port Single-Phase Solid-State Transformer in the Electromagnetic Timescale
}

\author{
Rui Wang ${ }^{1(\mathbb{D})}$, Qiuye Sun ${ }^{1,2, *}$, Qifu Cheng ${ }^{1,3}$ and Dazhong Ma ${ }^{1}$ \\ 1 College of Information Science and Engineering, Northeastern University, Shenyang 110004, China; \\ 1610232@stu.neu.edu.cn (R.W.); lowkeycheng@163.com (Q.C.); madazhong@ise.neu.edu.cn (D.M.) \\ 2 State Key Laboratory of Synthetical Automation for Process Industries, Northeastern University, \\ Shenyang 110004, China \\ 3 State Grid Shenyang Electric Power Supply Company, Shenyang 110004, China \\ * Correspondence: sunqiuye@ise.neu.edu.cn; Tel.: +86-139-9881-1006
}

Received: 8 August 2018; Accepted: 23 August 2018; Published: 27 August 2018

\begin{abstract}
This paper proposes an overall practical stability assessment for a multi-port single-phase solid-state transformer (MS3T) in the electromagnetic timescale. When multiple stable subsystems are combined into one MS3T, the newly formed MS3T has a certain possibility to be unstable. Thus, this paper discusses the stability assessment of the MS3T in detail. First and foremost, the structure of the MS3T and its three stage control strategies are proposed. Furthermore, the stability analysis of each of the MS3T's subsystems is achieved through the closed loop transfer function of each subsystem, respectively, including an AC-DC front-end side converter, dual active bridge (DAB) with a high-frequency (HF) or medium-frequency (MF) transformer, and back-end side incorporating DC-AC and dc-dc converters. Furthermore, the practical impedance stability criterion in the electromagnetic timescale, which only requires two current sensors and one external high-bandwidth small-signal sinusoidal perturbation current source, is proposed by the Gershgorin theorem and Kirchhoff laws. Finally, the overall stability assessment, based on a modified impedance criterion for the MS3T is investigated. The overall practical stability assessment of the MS3T can be validated through extensive simulation and hardware results.
\end{abstract}

Keywords: stability assessment; MS3T; perturbation approach; electromagnetic timescale

\section{Introduction}

With the high expansion of distributed renewable energy utilization, the Energy Internet (EI), the future renewable electric energy delivery and management (FREEDM) system, and microgrids have been widely researched [1-4]. Meanwhile, since the allowable weight and volume of a traction transformer are restricted, the solid-state transformer (SST) (also known as the Power Electronic Transformer, Energy Router, Intelligent Universal Transformer, etc.) has been one of the main elements in the EI, FREEDM, and microgrids [5]. Compared with traditional transformers, the SST has several advantages, such as low weight, low volume, unity power factor, fault isolation, bidirectional power flow capability, high controllability, etc. [6].

Obviously, stability is a basic necessity to ensure the performance of the system. If the system is unstable, it is of no value to research its performance [7-9]. Thus, the stability assessment of the multi-port single-phase solid-state transformer (MS3T) is a significant topic. As shown in Figure 1, from the viewpoint of the multi-timescale, the stability assessment can be divided into two parts [10], i.e., small-signal stability assessment and large-signal stability assessment. The non-linear behavior 
should be researched while the large-signal stability assessment is considered. Besides, this large-signal stability assessment pays more attention to the dynamic and transient events of the system. However, the small-signal stability assessment pays more attention to the steady state, which is studied in the electromagnetic timescale. According to the existing literature, small-signal stability assessment in the electromagnetic timescale always adopts a linearized method around a steady operating point [11-13]. Compared with traditional power systems, the small-signal stability assessment of the MS3T is a critical issue due to the low-inertia and negative-impedance nature of systems dominated by power electric converters [14]. Thus, this paper focuses on the small-signal stability assessment, which adopts a linearized method around a steady operating point, for the MS3T in the electromagnetic timescale.

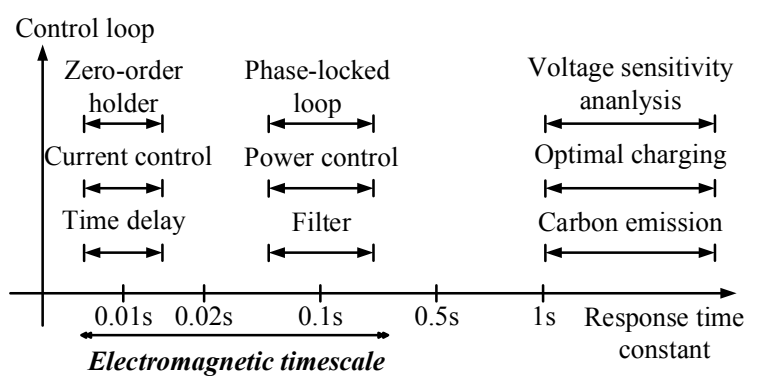

Figure 1. Extending the multi-timescale classification of a power electronic converter.

Traditionally, there are three stages in the MS3T, i.e., the AC-DC front-end side stage, dual active bridge DAB stage, and back-end side stage [15]. Although the three stages are able to achieve the key appealing features for MS3T together, the MS3T is highly subject to instabilities on account of the interactions among the three stages. The SST stability analysis strategy based on each subsystem model was first proposed by M. Khazraei [16]. Therein, each subsystem control strategy was so specialized that it was not easy for the common SST to utilize. Furthermore, the stability design criteria for a distribution system with SSTs were first proposed in References [17,18], and these stability criteria did not analyze the stability of the SST itself. Moreover, the premise of previous papers was that each subsystem of the SST was stable in the distribution system, and a practical method of stability analysis for the general SST was not proposed. Thus, the stability assessment of the SST in the electromagnetic timescale still remains a challenge that has rarely been researched.

Three main stability assessment methods were utilized to analyze the stability of the power electronics converter-dominated system in the electromagnetic timescale, i.e., a state-space-based approach, a transfer function approach, and an impedance-based approach. Compared with the state-space-based approach and transfer function approach, the impedance-based approach had several potential advantages to explicitly explain the impact of individual subsystems on the system stability [19]. Thus, the impedance-based approach should be applied to assess the stability of the MS3T. The impedance-based approach was first proposed by Middlebrook [19-24]. Furthermore, it is significant to check the right half-plane (RHP) poles of the transfer function matrix when using the generalized Nyquist criterion (GNC) for stability analysis. Several papers have been proposed to eliminate the step of checking the RHP poles, such as inverse generalized Nyquist criterion [21] and sum type criterion [22]. In order to reduce the computational complexity of the stability assessment, norm-based impedance methods were put forward one after another [23]. To deal with the bidirectional power flow, the sum type criterion was first proposed to assess the stability of the power electric converter [22]. Furthermore, according to the different applications of the impedance-based approach, Mohammad Amin et al. proposed a stability assessment for high-voltage DC (HVDC) systems [24], and an impedance-based local stability criterion was proposed to assess the stability of DC distributed power systems [25]. To sum up, it is advisable to apply the impedance-based approach to assess the stability of the MS3T. 
To date, the overall practical stability assessment of the MS3T in the electromagnetic timescale has not been proposed. Thus, the objective of this paper is to propose a practical impedance-based stability assessment of the MS3T. Compared with the previous existing literature, the proposed stability assessment has the following features:

(1) This paper researches the structure of the MS3T and its subsystem control strategy;

(2) The stability analysis of each of the MS3T's subsystems is achieved, such as the AC-DC front-end side converter, the DAB with an HF or MF transformer, and the back-end side incorporating DC-AC and DC-DC converters;

(3) The overall practical stability assessment in the electromagnetic timescale, based on the measured impedance stability analysis, for the MS3T is investigated. Furthermore, this stability assessment only requires two current sensors and one external high-bandwidth small-signal sinusoidal perturbation current source;

(4) The relative simulation and experiment results validate the performance of the stability assessment.

The rest of this paper is organized as follows. This paper establishes an MS3T structure model. Subsequently, each subsystem stability analysis of the MS3T is proposed in Section 2. Section 3 provides the overall stability assessment of the MS3T. In Sections 4 and 5, the proposed impedance-based assessment is validated through extensive simulation and hardware results. Finally, the conclusions are given in Section 6.

\section{MS3T Structure Model}

As shown in Figure 2, the MS3T structure model can be divided into four subsystems including an AC-DC front-end side converter subsystem [26], a DAB with an HF or MF transformer subsystem [27], a back-end side DC-AC converter subsystem [28], and a back-end side DC-DC converter subsystem [21]. $Z_{\text {out }, \text { sub } 1}, Z_{\text {in,sub } 2}, Z_{\text {out }, \text { sub } 2}, Z_{\text {in,sub3 }}$, and $Z_{\text {in,sub } 4}$ are the output impedances of subsystem 1, the input impedance of subsystem 2, the output impedance of subsystem 2 , the input impedance of subsystem 3 , and the input impedance of subsystem 4 , respectively. The AC-DC front-end side converter transforms the $220 \mathrm{~V}$ AC bus to a $400 \mathrm{~V}$ DC distribution bus. Furthermore, the DAB with an HF or MF transformer is used to reduce this DC voltage to a regulated DC voltage. Finally, the back-end side DC-AC converter produces an AC voltage to integrate an AC load, and the back-end side DC-DC converter produces a DC voltage to integrate a DC load [29]. As this paper focuses on the small-signal stability of the MS3T, slow control strategies, such as second-level coordinated control, optimal energy management, and so on, are ignored [30]. Therefore, the impedance-based stability approach can be applied to assess the overall stability of the MS3T. In light of the impedance-based approach, the system will be stable in the electromagnetic timescale if and only if the system satisfies following conditions: (1) each subsystem of the MS3T is stable; (2) the number of the counterclockwise encirclements of the $(-1, j 0)$ point by the ratio between the output source impedance and the input load impedance locus is equal to the number of the RHP poles of the minor loop gain. In reality, the second condition is usually judged only by checking whether the locus encircles the $(-1, j 0)$ point [21]. The small-signal models of each subsystem in the MS3T are researched as follows.

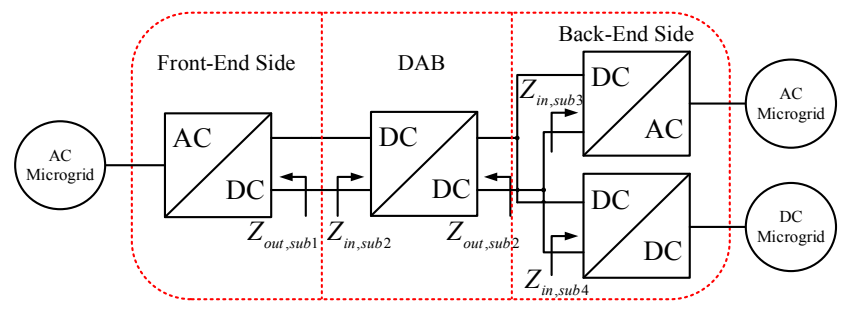

Figure 2. The multi-port single-phase solid-state transformer (MS3T) structure model with three stages. 


\subsection{The AC-DC Front-End Side Converter}

Both the control strategy and the topological structure are shown in Figure 3. There are four main controllers in the AC-DC front-end side converter, i.e., voltage controller with virtual inertia, DC voltage controller, DC current feedforward controller, and current controller. Thus, the mathematical expression of the converter is obtained as follows:

$$
\left\{\begin{array}{l}
u_{g d}=u_{a b d}+R_{r} i_{g d}+L_{r} \frac{d i_{g d}}{d t}+\omega L_{r} i_{g q} \\
u_{g q}=u_{a b q}+R_{r} i_{g q}+L_{r} \frac{d i_{g q}}{d t}-\omega L_{r} i_{g d}
\end{array}\right.
$$

The current controller is described as $G_{s u b 1 i}(s)=k_{\text {sub1ip }}+k_{\text {sublii }} / s$. Thus, Equation (1) can be rewritten as follows:

$$
\left\{\begin{array}{l}
u_{g d}=-G_{s u b 1 i}(s)\left(i_{g d}^{*}-i_{g d}\right)+u_{a b d}+\omega L_{r} i_{g q} \\
u_{g q}=-G_{s u b 1 i}(s)\left(i_{g q}^{*}-i_{g q}\right)+u_{a b q}-\omega L_{r} i_{g d}
\end{array}\right.
$$
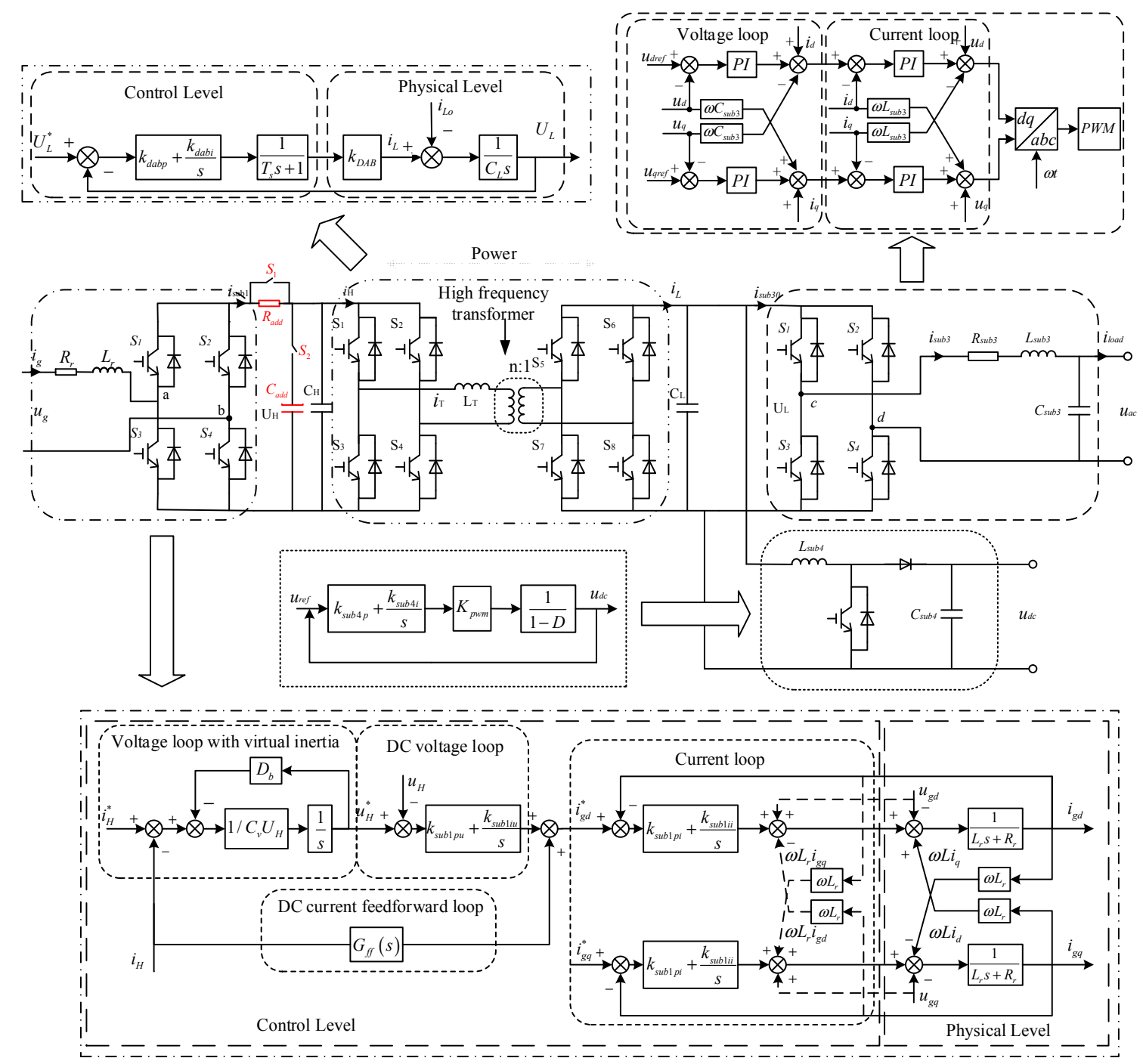

Figure 3. The overall control strategy and topological structure of the MS3T. 
Moreover, the small-signal model of the current controller can be shown as follows:

$$
\left\{\begin{array}{l}
i_{g d}(s)=\left(i_{g d}^{*}(s)-i_{g d}(s)\right) G_{s u b 1 i}(s) /\left(R_{r}+L_{r} s\right) \\
i_{g q}(s)=\left(i_{g q}^{*}(s)-i_{g q}(s)\right) G_{s u b 1 i}(s) /\left(R_{r}+L_{r} s\right)
\end{array}\right.
$$

Neglecting the energy loss, the following equation can be obtained due to the power balance between two sides of the converter.

$$
\frac{1}{2}\left(u_{g d} i_{g d}+u_{g q} i_{g q}\right)=u_{H} i_{s u b 1}=u_{H}\left(C \frac{d u_{H}}{d t}+i_{H}\right)
$$

Furthermore, both $U_{g d}$ and $I_{g d}$ are equivalent to zero in the steady state [26]. Thus, the small-perturbation Equation (5) can be obtained as follows:

$$
\frac{1}{2}\left(U_{g d} \Delta i_{g d}+\Delta u_{g d} I_{g d}\right)=C U_{H} \frac{d \Delta u_{H}}{d t}+U_{H} \Delta i_{H}+\Delta u_{H} I_{H}
$$

In light of the superposition theorem, the relations between $u_{H}(s)$ and $i_{g d}(s), u_{H}(s)$ and $i_{H}(s)$, respectively, are obtained as follows:

$$
\left\{\begin{array}{l}
G_{1}(s)=u_{H}(s) / i_{g d}(s)=U_{g d} / 2\left(C U_{H} s+I_{H}\right) \\
G_{2}(s)=u_{H}(s) / i_{H}(s)=-U_{H} /\left(C U_{H} s+I_{H}\right)
\end{array}\right.
$$

Neglecting the influence of the main grid voltage and virtual inertia controller, the DC output current feedforward controller and the voltage controller are applied, so the small-signal closed-loop transfer function can be obtained as follows:

$$
G_{c s u b 1}(s)=\frac{u_{H}(s)}{i_{H}(s)}=\frac{G_{v i r}(s) G_{s u b 1 v}(s) G_{c}(s) G_{1}(s)+G_{2}(s)}{1+G_{s u b 1 v}(s) G_{c}(s) G_{1}(s)}
$$

where $G_{\text {sub1v }}(s)=k_{\text {sublvp }}+k_{\text {sub1vi }} / s, G_{c}(s)=G_{\text {subli }}(s) /\left(G_{\text {subli }}(s)+\left(L_{r} s+R_{r}\right)\right), G_{\text {vir }}(s)=$ $-1 /\left(D_{b}+G_{\text {sublv }}(s) U_{n} s\right)$.

\subsection{DAB with an HF or MF Transformer}

As shown in Figure 3, the DAB is a bidirectional DC-DC power electrical converter with a phase-shift controller based on active bridges interfaced with a high/middle frequency transformer. Therefore, the expression of the transmitted power can be provided as follows:

$$
P_{H}=\frac{n U_{H} U_{L}}{4 L_{T}} d(1-d) T_{s}=U_{L} I_{L}
$$

Defining $D_{s}=d(1-d)$. Thus, Equation (8) can be rewritten as follows:

$$
\frac{n U_{H}}{4 L_{T}} T_{s} D_{s}=I_{L}
$$

Defining $k_{D A B}=I_{L} / D_{S}$, where $k_{D A B}=n U_{H} T_{S} / 4 L_{T}$. Using Faraday's law, the capacitor voltage can be calculated as:

$$
I_{L}-I_{L o}=C_{L} \frac{d U_{L}}{d t}
$$


The current controller is described as $G_{s u b 2}(s)=k_{s u b 2 p}+k_{s u b 2 i} / s$. The time delay is represented as $G_{d}(s)=1 /\left(T_{s} s+1\right)$. While a feedback loop (the feedback coefficient $\left.A=1 / k_{D A B}\right)$ is adopted in the $\mathrm{DAB}$, the small-signal closed-loop transfer function of the $\mathrm{DAB}$ is given as follows:

$$
G_{c s u b 2}(s)=\frac{G_{d}(s)-1}{k_{D A B} G_{s u b 2}(s) G_{d}(s)+C_{L} s}
$$

\subsection{The DC-AC or DC-DC Back-End Side Converter}

The DC-AC back-end side converter controlled by the traditional voltage/current double controller is shown in Figure 3. Thus, the current controller mathematical equations of the converter are represented by Equation (12).

$$
\left\{\begin{array}{l}
L_{s u b 3} \frac{d i_{\text {sub3d }}}{d t}+R_{\text {sub } 3} i_{\text {sub3d }}=u_{c d d}-u_{\text {sub3d }}+\omega L_{\text {sub } 3} i_{\text {sub3q }} \\
L_{\text {sub3 } 3} \frac{i_{\text {sub3q }}}{d t}+R_{\text {sub } 3} i_{\text {sub } 3 q}=u_{c d q}-u_{\text {sub3q }}-\omega L_{s u b 3} i_{\text {sub } 3 d}
\end{array}\right.
$$

The current controller can be described as $G_{\text {sub3i }}(s)=k_{\text {sub3ip }}+k_{\text {sub3ii }} / s$, and Equation (12) can be rewritten as:

$$
\left\{\begin{array}{l}
i_{\text {sub } 3 d}(s)=\frac{G_{\text {sub3i }}(s)\left(i_{\text {sub3d }}^{*}(s)-i_{\text {sub3d }}(s)\right)}{\left(R_{\text {sub }}+L_{\text {sub } 3 s} s\right)} \\
i_{\text {sub3q }}(s)=\frac{G_{\text {sub3i }}(s)\left(i_{\text {sub3 } 3 q}(s)-i_{\text {subb3q }}(s)\right)}{\left(R_{\text {sub } 3}+L_{\text {sub } 3} s\right)}
\end{array}\right.
$$

Moreover, when both the symmetrical characteristic and time delay are considered, the small-signal open-loop transfer function can be expressed as:

$$
G_{o i}(s)=\frac{G_{s u b 3 i}(s)\left(\frac{1}{1+T_{s} s}\right)\left(\frac{K_{p w m}}{1+0.5 T_{s} s}\right)}{\left(R_{s u b 3}+L_{s u b 3} s\right)}
$$

Therefore, the small-signal closed-loop transfer function can be provided as follows:

$$
G_{s u b 3 I}=\frac{G_{o i}(s)}{1+G_{o i}(s)}
$$

The similar analysis process for the voltage controller can be applied to that described previously to gain the voltage controller closed-loop transfer function. The voltage controller mathematical equations of the inverter are provided as follows:

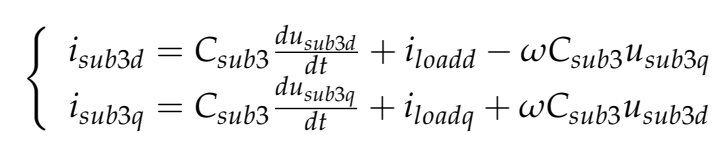

The current controller is described as $G_{\text {sub3v }}(s)=k_{\text {sub3vp }}+k_{\text {sub3vi }} / s$, and Equation (17) can be rewritten as follows:

$$
\left\{\begin{array}{l}
i_{\text {sub3d }}^{*}=G_{\text {sub3v }}(s)\left(u_{\text {sub3d }}^{*}(s)-u_{\text {sub3d }}(s)\right)-i_{\text {loadd }}-\omega C_{\text {sub } 3} u_{\text {sub3q }} \\
i_{\text {sub } 3 q}^{*}=G_{\text {sub3v }}(s)\left(u_{\text {sub3q }}^{*}(s)-u_{\text {sub } 3 q}(s)\right)+i_{\text {loadq }}+\omega C_{\text {sub } 3} u_{\text {sub3d }}
\end{array}\right.
$$

Finally, when both the time delay and the symmetrical characteristic are considered, the open-loop transfer function and closed-loop transfer function of the overall DC-AC converter can be expressed as follows:

$$
\left\{\begin{array}{c}
G_{\text {osub } 3}(s)=\frac{G_{\text {sub } 3 v}(s) G_{\text {sub3 } 3}(s)}{C_{\text {subbs }} s\left(1+T_{s} s\right)} \\
G_{\text {csub } 3}(s)=\frac{G_{\text {osub } 3}(s)}{1+G_{\text {osub } 3}(s)}
\end{array}\right.
$$


As seen in Figure 3, the DC-DC converter adopts the traditional PI closed control strategy, the voltage controller is described as $G_{s u b 4}(s)=k_{s u b 4 p}+k_{s u b 4 i} / s$. Thus, the closed-loop transfer function of the overall DC-DC converter is expressed as follows:

$$
G_{c s u b 4}(s)=\frac{G_{s u b 4}(s) K_{\text {pwom }}}{(1-D)+G_{s u b 4}(s) K_{\text {pwm }}}
$$

\section{The Stability Assessment of the Overall MS3T}

Based on the aforementioned analysis, a practical impedance-based stability assessment in the electromagnetic timescale needs to be proposed to assess the stability of the overall MS3T. As shown in Figure 4, the total input-to-output transfer function of the cascade of two individual stable subsystems, which are module 1 and module 2, is:

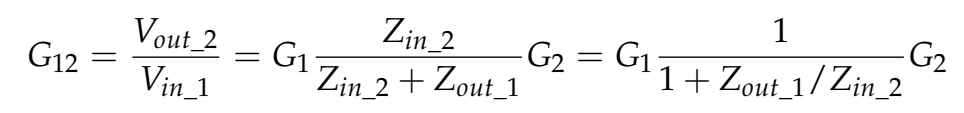

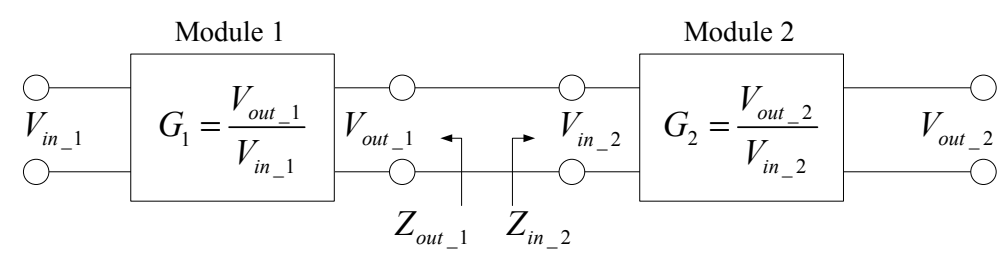

Figure 4. Interconnection of two stable independent systems.

Since $G_{1}$ and $G_{2}$ do not have the right half-plane pole, the system is stable if and only if $1 /\left(1+Z_{\text {out } \_1} / Z_{\text {in_2 } 2}\right)$ does not have the right pole. In other words, drawing the Nyquist plot of $Z_{\text {out } \_1} / Z_{\text {in } \_}$, the system is stable if and only if the number of times that the trajectory encircles the $(-1, j 0)$ point in the clockwise direction is zero. The "black-box" unterminated modeling of a DC two-port network has been an attractive topic widely utilized in engineering [31]. There is a demand in practice to acquire the linearized dynamics in the region "not far" from an operating point so as to assess the subsystem interaction and small-signal stability in the electromagnetic timescale. Apparently, the impedance of a general two-port network can be obtained by removing from the original environment and connecting to a high-bandwidth voltage source or current source.

As mentioned above, this ratio must not encircle the $(-1+j 0)$ point on Nyquist contours. Moreover, according to the Gershgorin theorem, if the eigenvalues of $Z_{\text {out_1 }} / Z_{\text {in_ } 2}$ at all frequencies are restrained inside the unit circle, the stability of the AC microgrids can be guaranteed, i.e.,:

$$
\left|Z_{\text {out_1 }} / Z_{\text {in_2 }}\right|<1
$$

Then, the necessary and sufficient condition satisfying Equation (22) is gained by Equation (21), which can be further transformed to Equation (23), since $V_{\text {out }} 1=V_{\text {in_ } 2}$.

$$
\begin{gathered}
\left|\frac{V_{\text {out_1 }} / i_{\text {out_1 }}}{V_{\text {in_2 }} / i_{\text {in__ }}}\right|<1 \\
\left|i_{\text {out_1 }}\right|>\left|i_{\text {in_2 }}\right|
\end{gathered}
$$

Moreover, according to the Kirchhoff laws, when the multiple paralleled subsystems are connected as in module $2, Z_{i n \_2}$ can be rewritten as follows:

$$
Z_{i n \_2}=Z_{i n, s u b 1} / / Z_{i n, s u b 2} / / \ldots / / Z_{i n, s u b n}
$$


Thus, Equation (23) can be rewritten as follows:

$$
\left|i_{\text {out } \_1}\right|>\left|i_{\text {in_2sub } 1}+\ldots+i_{\text {in_2subn }}\right|
$$

As shown in Figure 5, the stability of the MS3T can be assessed by an external high-bandwidth small-signal sinusoidal perturbation current $\hat{i}_{p}$, which is injected into the DC bus while the MS3T is in the steady state of the operating point. Furthermore, the small-signal response current in the source side and load side are measured. According to the aforementioned analysis, while the three stages are stable, the MS3T must be stable if Equations (26) and (27) are satisfied as follows:

$$
\begin{gathered}
\left|\Delta i_{\text {out } t \text { sub } 1}\right|>\left|\Delta i_{\text {in,sub } 2}\right| \\
\left|\Delta i_{\text {out }, \text { sub } 2}\right|>\left|\Delta i_{\text {in,sub } 3}+\Delta i_{\text {in,sub } 4}\right|
\end{gathered}
$$

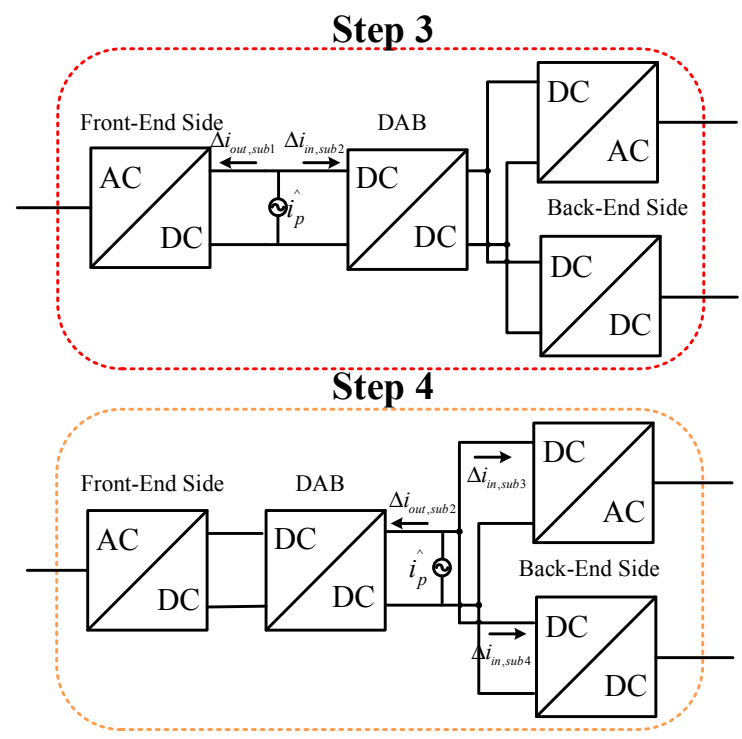

Figure 5. Setup for the terminal characterization of the MS3T.

Furthermore, it is also possible to only measure the source-side response current to assess the stability of the MS3T, since $\hat{i_{p}}=\Delta i_{\text {out,sub1 } 1}+\Delta i_{i n, s u b 2}$ and $\hat{i_{p}}=\Delta i_{\text {out }, \text { sub } 2}+\Delta i_{i n, s u b 3}+\Delta i_{i n, s u b 4}$ are satisfied. The sufficient condition at which the MS3T is stable is gained by Equations (28) and (29):

$$
\begin{gathered}
\left|\hat{i_{p}}\right|>2\left|\Delta i_{\text {in,sub2 }}\right| \\
\left|\hat{i_{p}}\right|>2\left|\Delta i_{\text {in,sub3 }}+\Delta i_{\text {in,sub4 }}\right|
\end{gathered}
$$

In summary, the proposed stability assessment based on impedance in the electromagnetic timescale, which is seen in Figure 6, can be described as follows:

Step1: Divide the overall MS3T into four subsystems containing an AC-DC front-end side converter, a DAB with an HF or MF transformer, a DC-AC back-end side converter, and a DC-DC back-end side converter, and derive the expressions of each subsystem closed-loop transfer function (Equations (7), (11), (18) and (19)).

Step2: Check the right half-plane pole of Equations (7), (11), (18) and (19). If the number of the right half-plane pole is zero, the assessment process continues to Step3. If not, the system is unstable. 
Step3: As shown in Figure 5, the small-signal response current of the input subsystem 2 side is measured. If the inequality (28) can be satisfied, the assessment process continues to Step4. If not, there is a possibility that the MS3T is unstable.

Step4: Similar to Step3, the small-signal response current in the input subsystem 3 side and input subsystem 4 side are measured. If the inequality (29) can be satisfied, the MS3T is stable. If not, there is a possibility that the MS3T is unstable.

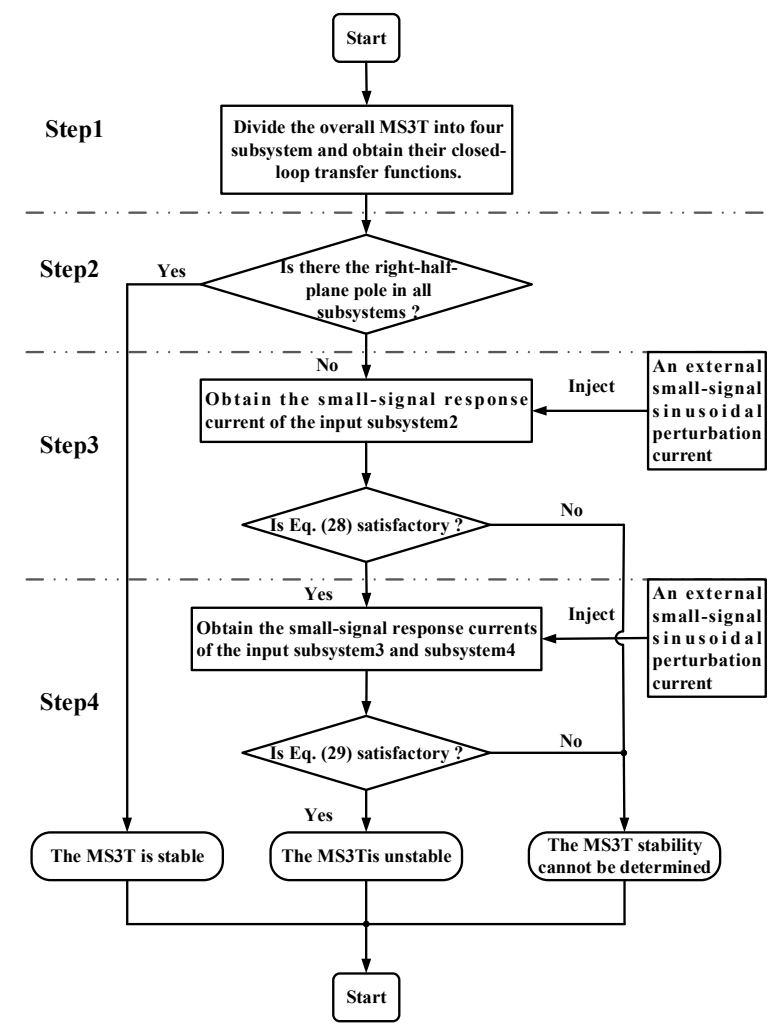

Figure 6. The flow chart of the proposed stability assessment method.

To sum up, the proposed stability assessment of the overall MS3T in the electromagnetic timescale is sufficient, but not necessary. Nevertheless, this stability assessment only requires two current sensors and one external high-bandwidth small-signal sinusoidal perturbation current source.

\section{Simulation Verification}

To verify the effectiveness of the proposed overall practical stability assessment for the MS3T in the electromagnetic timescale, simulations in MATLAB/Simulink were conducted for a $10 \mathrm{~kW} / 50$ $\mathrm{Hz}$ MS3T system. The system is depicted in Figure 3, and the parameters consisting of the AC-DC front-end side converter, DAB, and back-end side converters are shown in Tables 1-3, respectively. According to the closed transfer functions of each part of the MS3T in Section 2, each stage of the MS3T is stable if and only if the number of the right half-plane poles of each closed transfer function is zero. As shown in Table 4, where the poles reported can be obtained in MATLAB, there is no right half-plane pole for each stage of the closed transfer function. As a result, the three stages of the MS3T system are stable, and the stability of the overall MS3T depends on the interactions among the three stages. Of course, the 41 pole is very close to the imaginary axis in Table 4 . Thus, the stability margin of the DC-DC back-end side converter should be provided. In the s-plane, it is very convenient to represent the stability margin by using the distance from the ordinate to the left $\left(\sigma_{1}\right)$. According to the DC-DC 
back-end side converter subsystem closed-loop transfer function in Section 2 and the parameters of subsystem 4 in Table 3, the characteristic equation can be obtained as follows:

$$
3.2006 s+0.01212698=0
$$

Table 1. The parameters of the AC-DC front-end side converter.

\begin{tabular}{cc}
\hline Parameters & Values \\
\hline$G_{\text {sub1i }}(s)$ & $k_{\text {sub1ip }}=8, k_{\text {sub1ii }}=15$ \\
$G_{\text {sublv }}(s)$ & $k_{\text {sub1vp }}=1, k_{\text {sublvi }}=100$ \\
$C$ & $1 \mathrm{mF}$ \\
$U_{H}$ & $400 \mathrm{~V}$ \\
$I_{H}$ & $25 \mathrm{~A}$ \\
$U_{\text {gd }}$ & $311 \mathrm{~V}$ \\
$L_{r}$ & $3 \mathrm{mH}$ \\
$R_{r}$ & $0.01 \Omega$ \\
$D_{b}$ & 1 \\
$U_{n}^{\#}$ & $400 \mathrm{~V}$ \\
\hline
\end{tabular}

Table 2. The parameters of the dual active bridge (DAB).

\begin{tabular}{cc}
\hline Parameters & Values \\
\hline$G_{\text {sub } 2}(s)$ & $k_{\text {sub } 2 p}=1, k_{\text {sub } 2 i}=10$ \\
$T_{S}$ & $52.1 \mu \mathrm{s}$ \\
$D_{S}$ & 0.1875 \\
$I_{L}$ & $25 \mathrm{~A}$ \\
$C_{L}$ & $1 \mathrm{mF}$ \\
\hline
\end{tabular}

Table 3. The parameters of the AC-DC front-end side converter.

\begin{tabular}{cc}
\hline Parameters & Values \\
\hline$G_{\text {sub3v }}(s)$ & $k_{\text {sub3vp }}=0.0265, k_{\text {sub3vi }}=166.42$ \\
$G_{\text {sub } 3 i}(s)$ & $k_{\text {sub3ip }}=38.61, k_{\text {sub3ii }}=128.7$ \\
$L_{\text {sub3 }}$ & $3 \mathrm{mH}$ \\
$R_{\text {sub3 }}$ & $0.01 \Omega$ \\
$C_{\text {sub3 }}$ & $2.2 \mu \mathrm{F}$ \\
$T_{s}$ & $52.1 \mu \mathrm{s}$ \\
$k_{\text {pwm }}$ & 1.286 \\
$G_{\text {sub4 }}(s)$ & $k_{\text {sub } 4 p}=2.1, k_{\text {sub4i }}=9.43 \times 10^{-3}$ \\
$L_{\text {sub4 }}$ & $4.5 \mathrm{mH}$ \\
$C_{\text {sub4 }}$ & $1 \mathrm{mF}$ \\
$D$ & 0.5 \\
\hline
\end{tabular}

Table 4. The poles of the closed transfer functions.

\begin{tabular}{lclc}
\hline Poles & Values & Poles & Values \\
\hline$\lambda_{\text {sub } 11}$ & -100.0025 & $\lambda_{\text {sub } 12}$ & -1.8750 \\
$\lambda_{\text {sub13 }}$ & -114.7801 & $\lambda_{\text {sub14 }}$ & -409.3200 \\
$\lambda_{\text {sub15 }}$ & -2206.5249 & $\lambda_{\text {sub } 21}$ & -10.0008 \\
$\lambda_{\text {sub } 22}$ & $-9591.9+\mathrm{j} 49604$ & $\lambda_{\text {sub } 23}$ & $-9591.9-\mathrm{j} 49604$ \\
$\lambda_{\text {sub31 }}$ & -47560.9258 & $\lambda_{\text {sub32 }}$ & -18343.1446 \\
$\lambda_{\text {sub33 }}$ & $-5267.3217+\mathrm{j} 14609.2417$ & $\lambda_{\text {sub34 }}$ & $-5267.3217-\mathrm{j} 14609.2417$ \\
$\lambda_{\text {sub35 }}$ & $-168.3590+\mathrm{j} 2896.0036$ & $\lambda_{\text {sub36 }}$ & $-168.3590-\mathrm{j} 2896.0036$ \\
$\lambda_{\text {sub37 }}$ & -0.3333 & $\lambda_{\text {sub41 }}$ & $-3.7890 \times 10^{-3}$ \\
\hline
\end{tabular}


Substitute $s=z-\sigma_{1}$ into the above equation. Thus, the stability margin $\left(\sigma_{1}\right)$ can be obtained as follows:

$$
3.2006\left(z-\sigma_{1}\right)+0.01212698=0 \Rightarrow \sigma_{1}=-3.7890 * 10^{-3}
$$

From the viewpoint of the improvement of the stability margin using the proposed stability assessment, the stability margin $\left(\sigma_{1}\right)$ of the DC-DC back-end side converter can be presented as follows:

$$
\sigma_{1}=\frac{k_{\text {sub4i }} k_{p w m}}{k_{\text {sub } 4 p} k_{p w m}+(1-D)}
$$

Since $k_{p w m}$ and $D$ are always constant, the stability margin can be enhanced by increasing the ratio of $k_{\text {subui }}$ and $k_{\text {sub4p }}$.

Of course, with increasing of the order of the closed-loop transfer function, it is difficult to obtain the stability margin $\left(\sigma_{1}\right)$. Phase Margin (PM) and Gain Margin (GM) can become an advisable choice. The Bode diagram of the DC-AC back-end side converter can be seen in Figure 7 as follows:

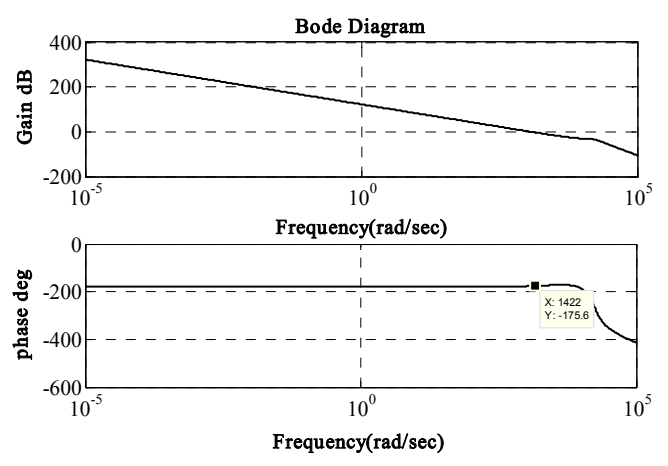

Figure 7. The Bode diagram of the DC-AC back-end side converter.

Thus, the Phase Margin of the DC-AC back-end side converter is $4.4^{\circ}$, and stability margin can also be improved by adjusting the voltage/current controller parameters.

\subsection{Stable Case}

In this section, the effectiveness of the proposed overall stability assessment for the MS3T can be verified. The stage system parameters are shown in Tables 1-3. The control strategy and the topological structure of the simulation text system are shown in Figure 3. Therein, the switch 1 (S1) is closed, and the switch 2 (S2) is opened. According to the aforementioned analysis in Section 3 and Table 4, the four subsystems of the MS3T are stable. Thus, the stability of the MS3T depends on the interaction among three stages. As shown in Figures 8 and 9, Step 3 and Step 4 in Section 3 are satisfied, so the MS3T should be stable. The stability of the MS3T can be directly verified by checking the waveform in the time domain. Apparently, the MS3T is stable, as shown in Figures 10-12, where the input voltage/current of the AC-DC front-end side converter, the output voltage/current of the DC-AC back-end side converter, and the output voltage/current of the DC-DC back-end side converter coincide with the stable phenomenon. Therefore, the effectiveness of the proposed stability assessment is verified. 


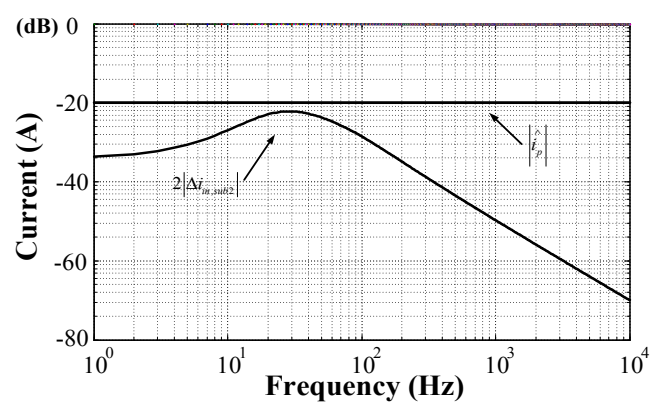

Figure 8. Relationship between measured currents of $\left|\hat{i_{p}}\right|$ and $2\left|\Delta i_{i n, s u b 2}\right|$ in a stable case.

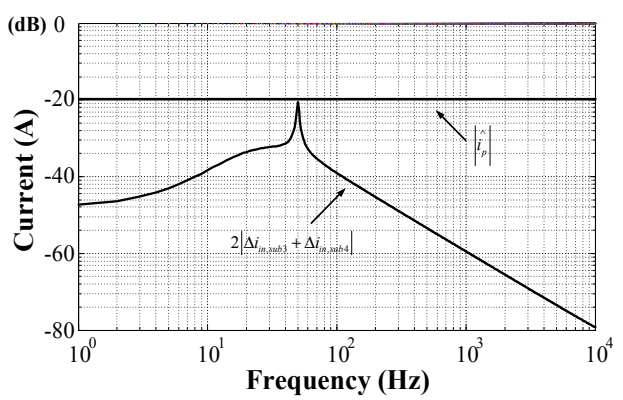

Figure 9. Relationship between measured currents of $\left|\hat{i_{p}}\right|$ and $2\left|\Delta i_{\text {in,sub3 }}+\Delta i_{\text {in,sub4 }}\right|$ in a stable case.

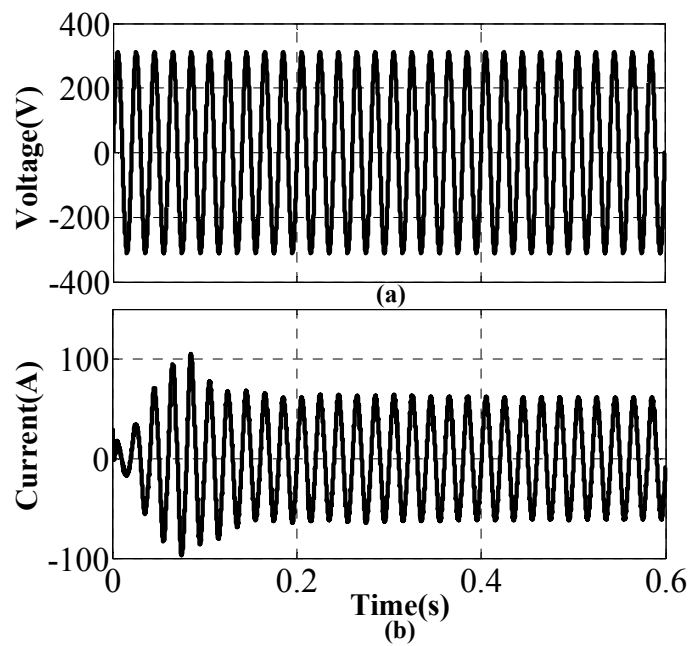

Figure 10. The input voltage/current of the AC-DC front-end side converter in a stable case. 


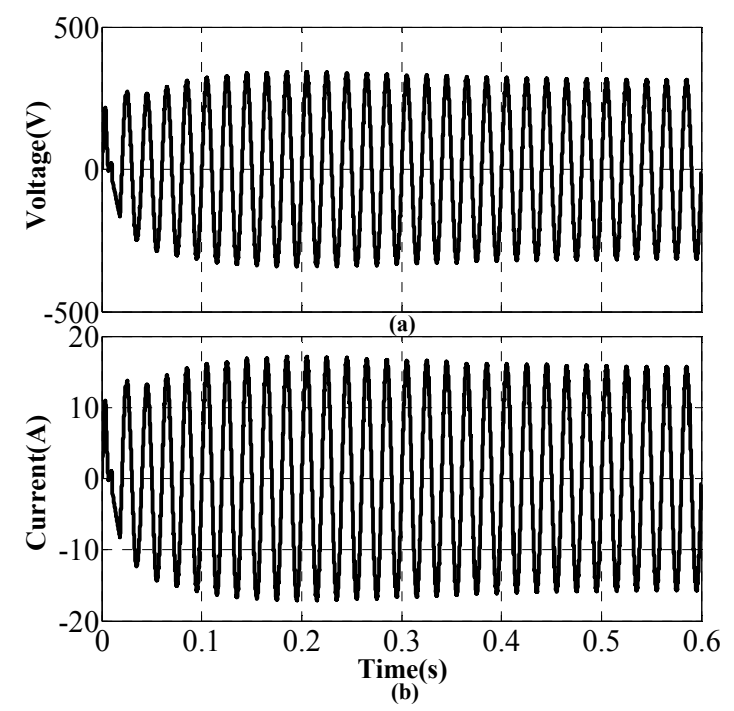

Figure 11. The output voltage/current of the DC-AC back-end side converter in a stable case.

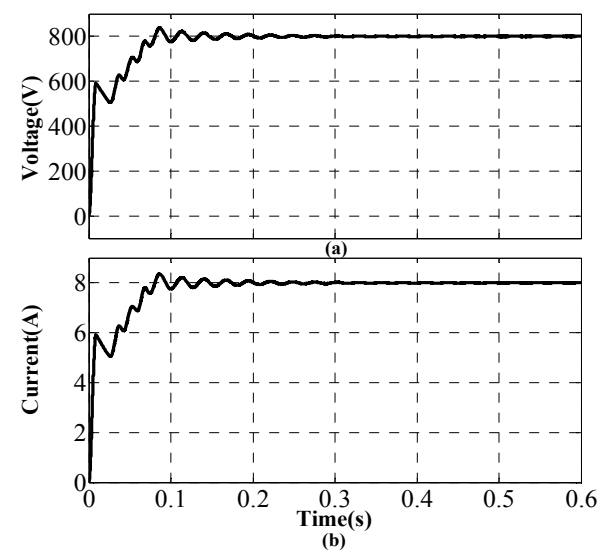

Figure 12. The output voltage/current of the DC-DC back-end side converter in a stable case.

Although the proposed stability assessment in this paper is not suitable for analyzing dynamic/transient processes, when the load changes, it can be applied to assess the stability of the MS3T in the next steady-state operation point. A case in point is that the output DC load of the MS3T changes from $R=100 \Omega$ to $R=50 \Omega$, and the other parameters are same as those in Tables 1-3. Since each controller of each subsystem is the same as that in the above simulation test system, the eigenvalues are also shown in Table 4. Thus, the stability of the MS3T depends on the interaction among the three stages. As shown in Figures 13 and 14, Step3 and Step 4 in Section 3 are satisfied, so the MS3T should be stable. The stability of the MS3T can be directly verified by checking the waveform in the time domain. Apparently, the MS3T is stable, as shown in Figures 15-17, where the input voltage/current of the AC-DC front-end side converter, the output voltage/current of the DC-AC back-end side converter, and the output voltage/current of the DC-DC back-end side converter coincide with the stable phenomenon. Thus, the performance of the proposed stability assessment can be verified. 


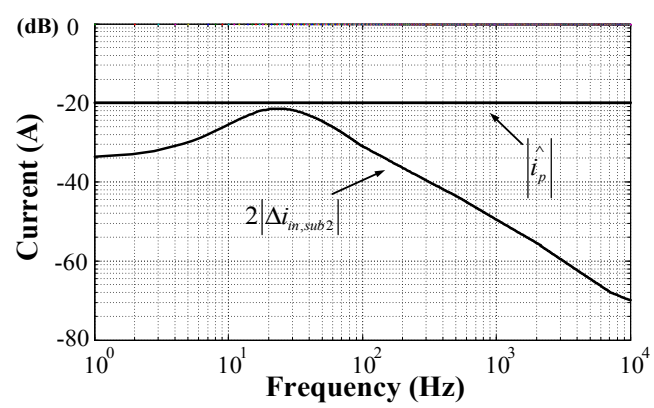

Figure 13. Relationship between measured currents of $\left|\hat{i}_{p}\right|$ and $2\left|\Delta i_{i n, s u b 2}\right|$ in the next steady-state operation point.

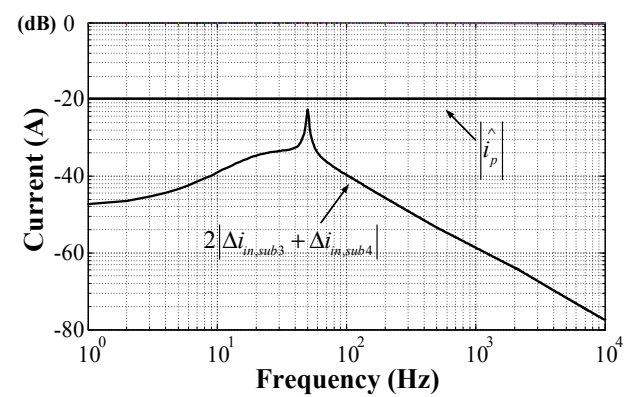

Figure 14. Relationship between measured currents of $\left|\hat{i_{p}}\right|$ and $2\left|\Delta i_{i n, s u b 3}+\Delta i_{i n, s u b 4}\right|$ in the next steady-state operation point.

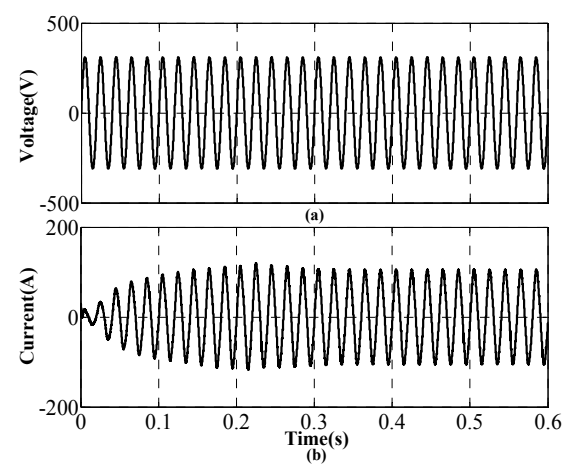

Figure 15. The input voltage/current of the AC-DC front-end side converter in the next steady-state operation point.

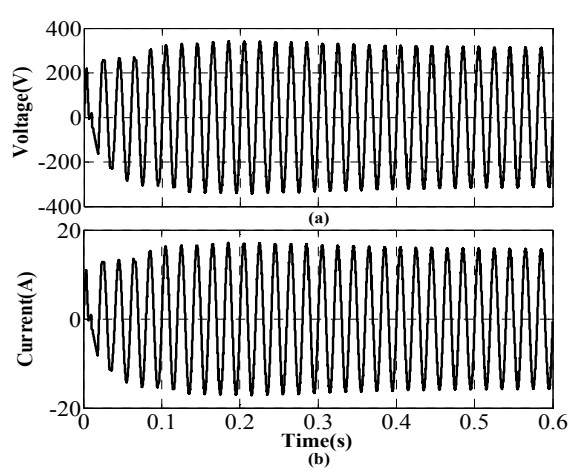

Figure 16. The output voltage/current of the DC-AC back-end side converter in the next steady-state operation point. 


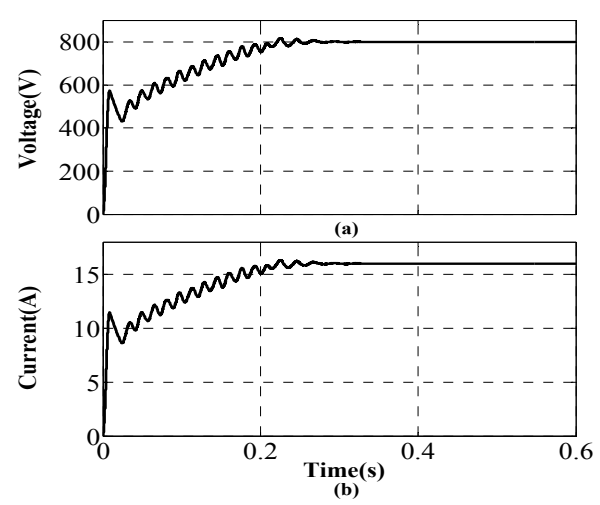

Figure 17. The output voltage/current of the DC-DC back-end side converter in the next steady-state operation point.

\subsection{Unstable Case}

In this section, the effectiveness of the proposed overall stability assessment for the MS3T in the electromagnetic timescale can be verified. The system parameters of each stage are also shown in Tables 1-3. The stability margin will be reduced by adding the impedance of the output source subsystem [23]. Thus, the control strategy and the topological structure of the simulation text system are shown in Figure 3. Therein, switch 1 (S1) is opened, switch 2 (S2) is closed, and $R_{a d d}=1.7 \Omega$ as well as $C_{a d d}=24 \mu \mathrm{F}$. Since $R_{a d d}$ and $C_{a d d}$ are only added in output side of the AC-DC front-end side converter, the closed-loop transfer functions of each subsystem are not affected, and each closed-loop transfer function in an unstable case is same with that in a stable case. Furthermore, the eigenvalues for the unstable case are same with those for stable case, which are shown in Table 4. Thus, each subsystem is stable. Moreover, Step3 in the Section 3 is not satisfied, as shown in Figure 18. Thus, Step4 is not executed. There is a possible that the MS3T is unstable. The stability of the MS3T can be directly verified by checking the waveform in the time domain. Apparently, the MS3T is unstable, as shown in Figures 19 and 20, where the input voltage/current of the AC-DC front-end side converter and the output voltage/current of the DAB coincide with the unstable phenomenon. To sum up, the MS3T is highly subject to instabilities on account of the interaction among the four subsystems, although each subsystem is stable. In other words, the instability of MS3T is caused by the interaction between subsystems. Therefore, the effectiveness of the proposed stability assessment is verified.

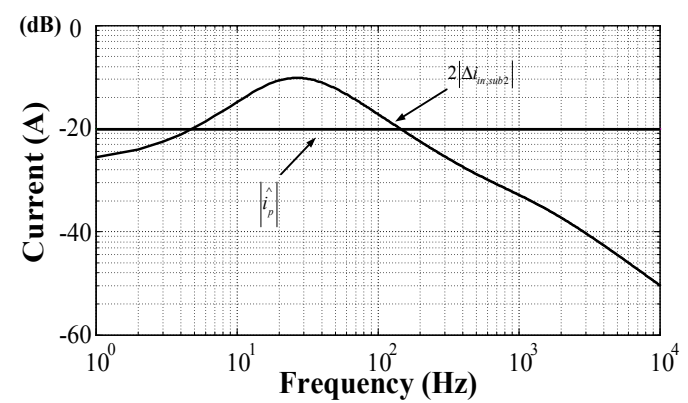

Figure 18. Relationship between measured currents of $\left|\hat{i_{p}}\right|$ and $2\left|\Delta i_{i n, s u b 3}+\Delta i_{i n, s u b 4}\right|$ in an unstable case. 


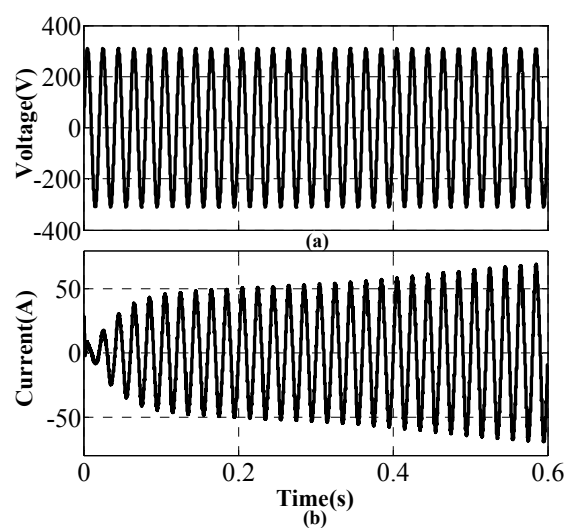

Figure 19. The input voltage/current of the AC-DC front-end side converter in an unstable case.

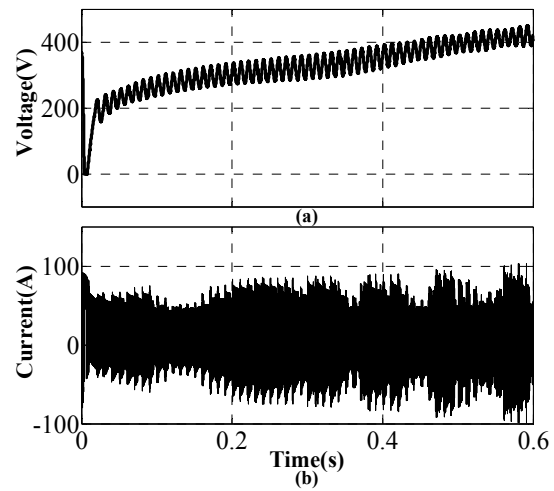

Figure 20. The output voltage/current of the DAB in an unstable case.

\section{Experimental Results}

A $220 \mathrm{~V}, 50 \mathrm{~Hz}$ MS3T was built to verify the effectiveness of the proposed approach. As shown in Figure 21, the whole platform of the MS3T is controlled by TMS320F28335DSP + XC6SLX9FPGA. Furthermore, the communication circuit is $R S 232 \& R S 485$, and the system switch frequency is $19.2 \mathrm{kHz}$. The model of the IGBTs in the AC-DC front-end side stage and back-end side stage is $2 M B I 75 U 4 A-120$, and the model of the IGBTs in the DAB stage is CM400DU-24NFH. The system controller parameters are the same as those in Section 4, and the other parameters are shown in Table 5. As shown in Table 6, there is no right half-plane pole for each stage of the closed transfer function. As a result, the three stages of the MS3T system are stable, and the stability of the overall MS3T depends on the interactions among the three stages.

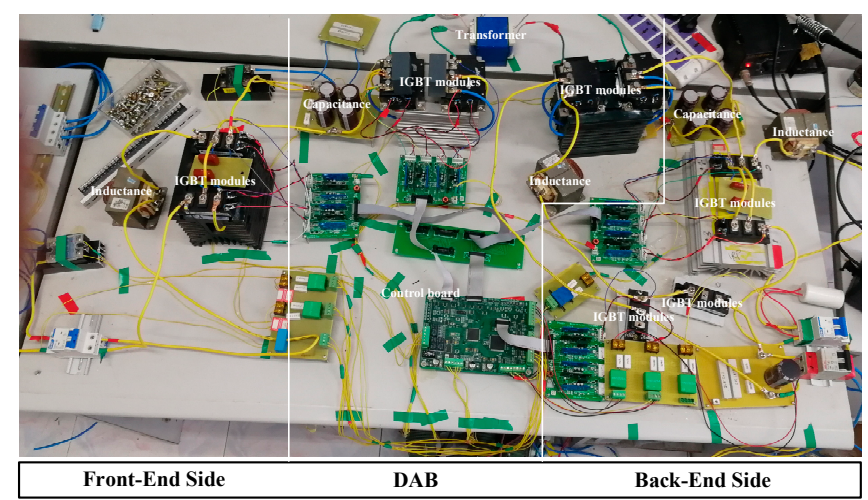

Figure 21. Experimental hardware test setup. 
Table 5. The experimental parameters of the MS3T.

\begin{tabular}{cccc}
\hline Parameters & Values & Parameters & Values \\
\hline$C$ & $1 \mathrm{mF}$ & $L_{r}$ & $2.5 \mathrm{mH}$ \\
$R_{r}$ & $0.01 \Omega$ & $C_{L}$ & $1 \mathrm{mF}$ \\
$L_{\text {sub3 } 3}$ & $2.5 \mathrm{mH}$ & $R_{\text {sub } 3}$ & $1 \mathrm{mF}$ \\
$C_{\text {sub } 3}$ & $12 \mu \mathrm{F}$ & $T_{S}$ & $52.1 \mu \mathrm{s}$ \\
$L_{\text {sub } 4}$ & $2.5 \mathrm{mH}$ & $C_{\text {sub } 4}$ & $1 \mathrm{mF}$ \\
\hline
\end{tabular}

Table 6. The poles of the experimental text system closed transfer functions.

\begin{tabular}{cccc}
\hline Poles & Values & Poles & Values \\
\hline$\lambda_{\text {sub } 11}$ & -100.0025 & $\lambda_{\text {sub } 12}$ & 1.8750 \\
$\lambda_{\text {sub } 13}$ & -114.9710 & $\lambda_{\text {sub } 14}$ & -392.4269 \\
$\lambda_{\text {sub } 15}$ & -2757.2272 & $\lambda_{\text {sub } 21}$ & -10.0008 \\
$\lambda_{\text {sub } 22}$ & $-9592+\mathrm{j} 49604$ & $\lambda_{\text {sub } 23}$ & $-95912-\mathrm{j} 49604$ \\
$\lambda_{\text {sub31 }}$ & -48634.9072 & $\lambda_{\text {sub32 }}$ & -19044.1187 \\
$\lambda_{\text {sub33 }}$ & $-4510.6015+\mathrm{j} 16665.5791$ & $\lambda_{\text {sub34 }}$ & $-4510.6015-\mathrm{j} 16665.5791$ \\
$\lambda_{\text {sub35 }}$ & $-39.4247+\mathrm{j} 1187.1495$ & $\lambda_{\text {sub36 }}$ & $-39.4247-\mathrm{j} 1187.1495$ \\
$\lambda_{\text {sub37 }}$ & -0.3333 & $\lambda_{\text {sub } 41}$ & $-3.7890 \times 10^{-3}$ \\
\hline
\end{tabular}

\subsection{Stable Case of the Experimental Test System}

In this section, the effectiveness of the proposed overall stability assessment for the MS3T is verified. According to the aforementioned analysis in Section 3 and Table 6, the four subsystems of the MS3T are stable. Thus, the stability of the MS3T depends on the interactions among the three stages. As shown in Figures 22 and 23, Step3 and Step 4 in Section 3 are satisfied, so the MS3T should be stable. The stability of the MS3T can be directly verified by checking the waveform in the time domain. Apparently, the MS3T is stable, as shown in Figures 24-26, where the input voltage of the AC-DC front-end side converter, the output voltage of the DC-AC back-end side converter, and the output voltage of the DC-DC back-end side converter coincide with the stable phenomenon. Therefore, the effectiveness of the proposed stability assessment is verified.

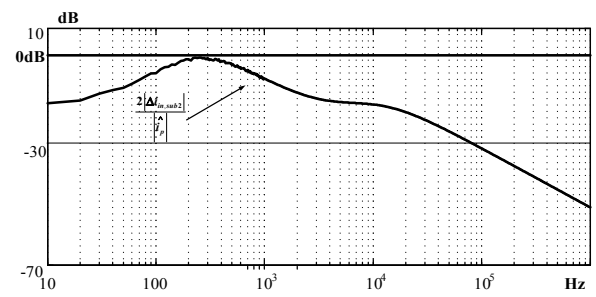

Figure 22. Relationship between measured currents of $\left|i_{p}\right|$ and $2\left|\Delta i_{i n, s u b 2}\right|$ in a stable case of the experiment test system.

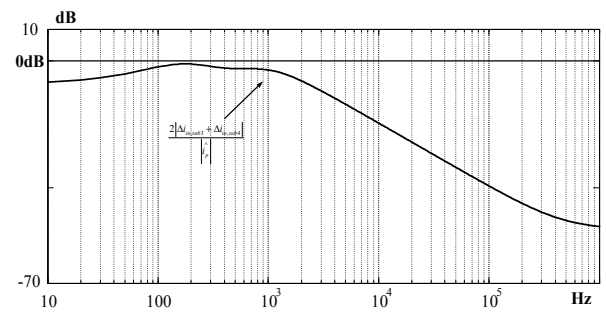

Figure 23. Relationship between measured currents of $\left|\hat{i_{p}}\right|$ and $2\left|\Delta i_{i n, s u b 3}+\Delta i_{\text {in,sub4 }}\right|$ in a stable case of the experiment test system. 


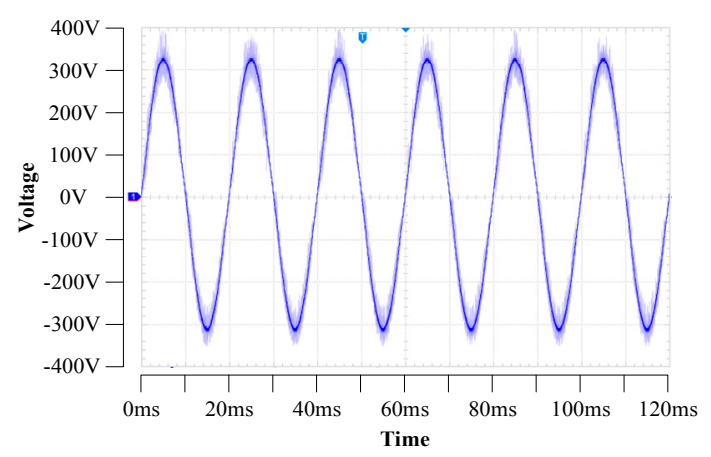

Figure 24. The input voltage of the AC-DC front-end side converter in a stable case of the experiment test system.

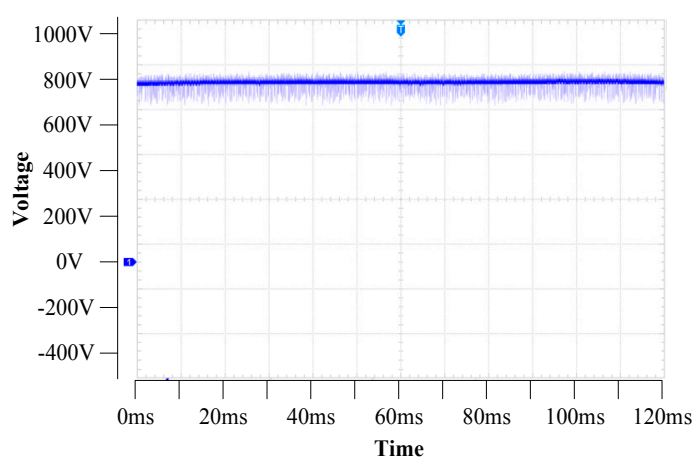

Figure 25. The output voltage of the DC-AC back-end side converter in a stable case of the experiment test system.

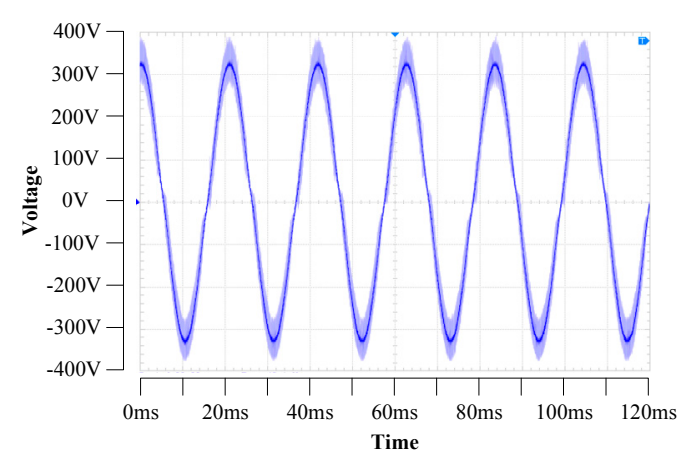

Figure 26. The output voltage of the DC-DC back-end side converter in a stable case of the experiment test system.

\subsection{Unstable Case of the Experimental Test System}

In order to verify the effectiveness of the proposed overall stability assessment for the MS3T, $R_{a d d}$ and $C_{a d d}$ were added in the output side of the AC-DC front-end side converter, which is same as that in the unstable case of Section 4 . Therein, $R_{a d d}=1.7 \Omega$ and $C_{a d d}=1000 \mu \mathrm{F}$. According to the aforementioned analysis in Section 3, Since $R_{a d d}$ and $C_{a d d}$ are only added in the output side of the AC-DC front-end side converter, the closed-loop transfer functions of each subsystem are not affected, and each closed-loop transfer function in the unstable case is same as that in the stable case. Furthermore, the eigenvalues for the unstable case are same as those for stable case, which are shown in Table 6. Moreover, the four subsystems of the MS3T are stable. Thus, the stability of the MS3T depends on the interactions among the three stages. As shown in Figure 27, Step3 in Section 3 is not satisfied, so there is a possibility that the MS3T is unstable. The stability of the MS3T can be directly verified by 
checking the waveform in the time domain. Apparently, the MS3T is unstable, as shown in Figures 28 and 29, where the input voltage of the AC-DC front-end side converter and the output voltage of the $\mathrm{DAB}$ coincide with the unstable phenomenon. Therefore, the effectiveness of the proposed stability assessment is verified.

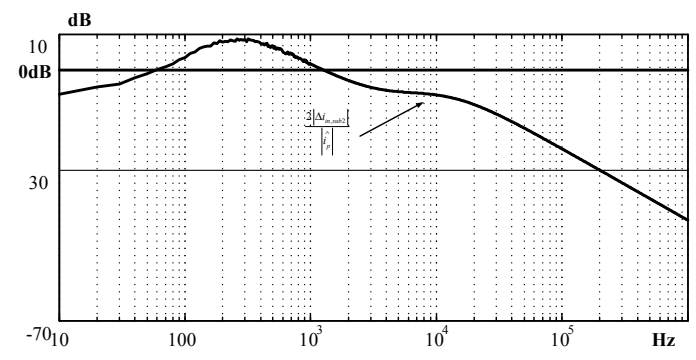

Figure 27. Relationship between measured currents of $\left|\hat{i}_{p}\right|$ and $2\left|\Delta i_{i n, s u b 2}\right|$ in an unstable case of the experiment test system.

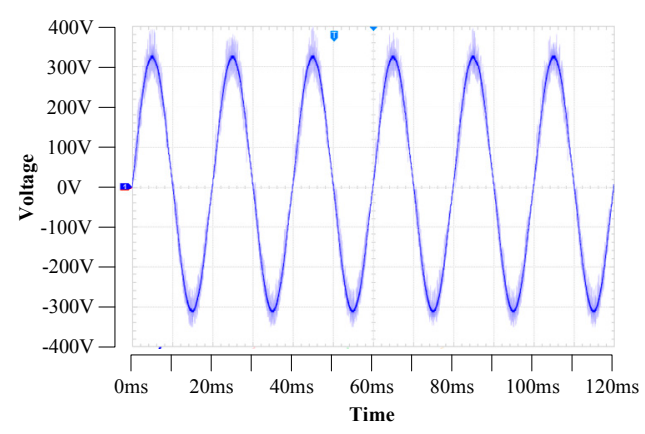

Figure 28. The input voltage of the AC-DC front-end side converter in an unstable case of the experiment test system.

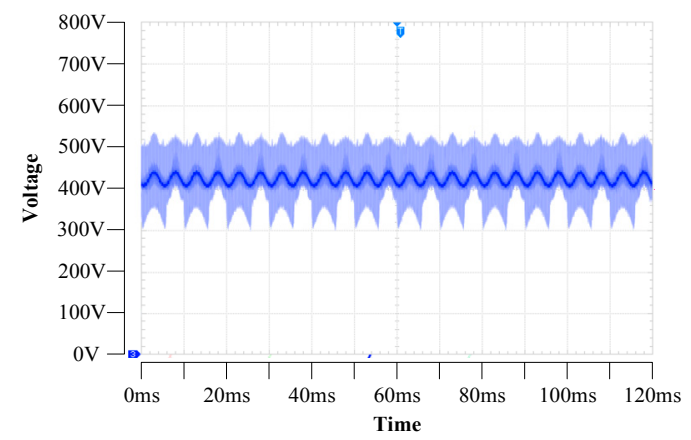

Figure 29. The output voltage of the DAB in an unstable case of the experiment test system.

\section{Conclusions}

With the high expansion of distributed renewable energy utilization, the MS3T has been widely applied. Meanwhile, the stability of the MS3T in an electromagnetic timescale is a basic requirement to ensure the performance of the MS3T. Thus, this paper proposed a practical stability assessment based on the impedance method for the MS3T in an electromagnetic timescale. First of all, the control strategy of the MS3T was investigated, and the stability analysis of each of its subsystems was researched. According to the small-signal method, the transfer function of each subsystem was built, and the stability of each subsystem was distinguished by the eigenvalue. Furthermore, the modified impedance stability criterion in the electromagnetic timescale was proposed by the Gershgorin theorem and Kirchhoff laws. Eventually, the stability assessment of the overall MS3T was 
verified through the simulation and experiment. Compared with the existing literature, this paper researched the structure of the MS3T and its subsystem control strategy, and the stability analysis of each of the MS3T's subsystems was achieved. Furthermore, the overall practical stability assessment in the electromagnetic timescale, based on measured impedance stability analysis, for the MS3T was investigated. Moreover, this stability assessment only requires two current sensors and one external high-bandwidth small-signal sinusoidal perturbation current source.

Author Contributions: All the authors conceived and designed the study. R.W. performed the simulation and wrote the manuscript with guidance from Q.S. and Q.C. Q.S., Q.C. and D.M. reviewed the manuscript and provided valuable suggestions.

Acknowledgments: This work was supported by the National Natural Science Foundation of China (NSFC) under Grant No. 61433004, No. 61573094, and No. 61773109.

Conflicts of Interest: The authors declare no conflict of interest.

\section{Nomenclature}

The AC-DC front-end side converter:

$C_{H} \quad$ dc-link output capacitor $(\mu \mathrm{F})$

$R_{r} \quad$ ac-link equivalent series resistance $(\Omega)$

$i_{g} \quad$ utility grid current (A)

$u_{g} \quad$ ac-link input filter inductor $(\mathrm{mH})$

$C_{v} \quad$ introduced virtual capacitance $(\mu \mathrm{F})$

$i_{H} \quad$ dc-link output current (A)

$G_{s u b 1 v}(s) \quad$ voltage controller

$L_{r} \quad$ ac-link input filter inductor $(\mathrm{mH})$

$D_{b} \quad$ droop coefficient

$U_{H} \quad$ dc-link voltage $(\mathrm{V})$

$U_{n} \quad$ rated dc bus voltage $(\mathrm{V})$

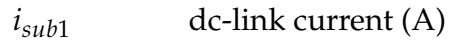

$\mathrm{G}_{\text {sub1i }}(s) \quad$ current controller

$D A B$ :

$P_{H} \quad$ transmitted power $(\mathrm{W})$

$U_{L} \quad$ output dc-link voltage (V)

d phase shift

$I_{L} \quad$ output dc-link current (A)

$I_{L 0} \quad$ output dc-link current in steady state

$G_{d}(s) \quad$ time delay ( $\left.\mu s\right)$

$n \quad$ turn ratio of the transformer

$L_{T} \quad$ equivalent leakage inductance $(\mathrm{mH})$

$T_{S} \quad$ half of the switching period $(\mu \mathrm{s})$

$C_{L} \quad$ output dc-link capacitor $(\mu \mathrm{F})$

$G_{\text {sub2 }}(s) \quad$ current controller

A feedback coefficient

The DC-AC or dc-dc back-end side converter:

$L_{s u b 3} \quad$ ac-link inductance $(\mathrm{mH})$

$R_{\text {sub3 } 3} \quad$ equivalent ac-link resistance $(\Omega)$

$u_{\text {sub3 } 3} \quad$ output ac-link voltage (V)

$K_{\text {pwm }} \quad$ converter gain

$G_{\text {sub3i }}(s) \quad$ current controller

$L_{\text {sub4 }} \quad$ dc-side input inductance $(\mathrm{mH})$

$G_{\text {sub4 }}(s) \quad$ PI controller

$C_{\text {sub3 } 3}$ ac-link capacitor $(\mu \mathrm{F})$

$i_{\text {sub3 }} \quad$ output ac-link current (A)

$u_{c d} \quad$ dc-link voltage $(\mathrm{V})$ 


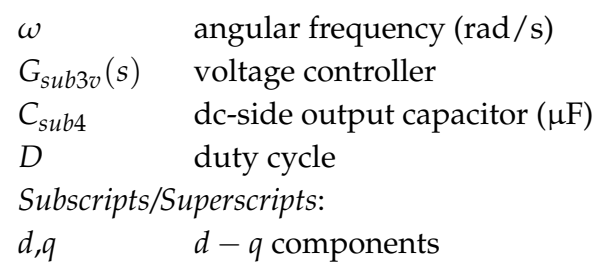

\section{References}

1. Wang, Z.; Huang, Z.; Song, C.; Zhang, H. Multiscale adaptive fault diagnosis based on signal symmetry reconstitution preprocessing for microgrid inverter under changing load condition. IEEE Trans. Smart Grid 2018, 9, 797-806. [CrossRef]

2. Zhang, H.; Li, Y.; Gao, D.W.; Zhou, J. Distributed optimal energy management for energy internet. IEEE Trans. Ind. Inform. 2017, 13, 3081-3097. [CrossRef]

3. Sun, Q.; Huang, B.; Li, D.; Ma, D.; Zhang, Y. Optimal placement of energy storage devices in microgrids via structure preserving energy function. IEEE Trans. Ind. Inform. 2016, 12, 1166-1179. [CrossRef]

4. Huang, Z.; Wang, Z.; Zhang, H. A diagnosis algorithm for multiple open-circuited faults of microgrid inverters based on main fault component analysis. IEEE Trans. Energy Conver. 2018, in press. [CrossRef]

5. Yun, H.-J.; Kim, H.-S.; Kim, M.; Baek, J.-W.; Kim, H.-J. A dab converter with common-point-connected winding transformers suitable for a single-phase 5-level SST system. Energies 2018, 11, 928. [CrossRef]

6. Ge, J.; Zhao, Z.; Yuan, L.; Lu, T. Energy feed-forward and direct feed-forward control for solid-state transformer. IEEE Trans. Power Electron. 2015, 30, 4042-4047. [CrossRef]

7. Zhang, H.; Liu, Z.; Huang, G.; Wang, Z. Novel weighting-delay-based stability criteria for recurrent neural networks with time-varying delay. IEEE Trans. Neural Netw. 2010, 21, 91-106. [CrossRef] [PubMed]

8. Zhang, H.; Wang, Z.; Liu, D. A comprehensive review of stability analysis of continuous-time recurrent neural networks. IEEE Trans. Neural Netw. 2014, 25, 1229-1262. [CrossRef]

9. Shen, P.; Guan, L.; Huang, Z.; Wu, L.; Jiang, Z. Active-Current control of large-scale wind turbines for power system transient stability improvement based on perturbation estimation approach. Energies 2018, 11, 1995. [CrossRef]

10. Yuan, H.; Yuan, X.; Hu, J. Modeling of grid-connected VSCs for power system small-signal stability analysis in DC-Link voltage control timescale. IEEE Trans. Power Syst. 2017, 32, 3981-3991. [CrossRef]

11. Rasheduzzaman, M.; Mueller, J.A.; Kimball, J.W. Reduced-Order Small-Signal Model of Microgrid Systems. IEEE Trans. Sustain. Energy 2015, 6, 1292-1305. [CrossRef]

12. Amin, M.; Molinas, M. Small-Signal Stability Assessment of Power Electronics Based Power Systems: A Discussion of Impedance- and Eigenvalue-Based Methods. IEEE Trans. Ind. Appl. 2017, 53, 5014-5030. [CrossRef]

13. Cao, W.; Ma, Y.; Yang, L.; Wang, F.; Tolbert, L.M. D-Q Impedance Based Stability Analysis and Parameter Design of Three-Phase Inverter-Based AC Power Systems. IEEE Trans. Ind. Electron. 2017, 64, 6017-6028. [CrossRef]

14. Kahrobaeian, A.; Mohamed, Y.A.R.I. Analysis and Mitigation of Low-Frequency Instabilities in Autonomous Medium-Voltage Converter-Based Microgrids with Dynamic Loads. IEEE Trans. Ind. Electron. 2014, 61, 1643-1658. [CrossRef]

15. Chen, H.; Divan, D. Design of a $10 \mathrm{kV}$ A Soft-Switching Solid State Transformer (S4T). IEEE Trans. Power Electron. 2018, in press.

16. Khazraei, M.; Prabhala, V.A.K.; Ahmadi, R.; Ferdowsi, M. Solid-state transformer stability and control considerations. In Proceedings of the 2014 IEEE Applied Power Electronics Conference and Exposition, Fort Worth, TX, USA, 16-20 March 2014; pp. 2237-2244.

17. Shah, D.G.; Crow, M.L. Stability Design Criteria for Distribution Systems with Solid-State Transformers. IEEE Trans. Power Deliv. 2014, 29. [CrossRef]

18. Shah, D.G.; Crow, M.L. Stability Assessment Extensions for Single-Phase Distribution Solid-State Transformers. IEEE Trans. Power Deliv. 2015, 30, 1636-1638. [CrossRef] 
19. Radwan, A.A.A.; Mohamed, Y.A.R.I. Analysis and Active-Impedance-Based Stabilization of Voltage-Source-Rectifier Loads in Grid-Connected and Isolated Microgrid Applications. IEEE Trans. Sustain. Energy 2013, 4, 563-576. [CrossRef]

20. Liu, J.; Feng, X.; Lee, F.C.; Borojevich, D. Stability margin monitoring for DC distributed power systems via perturbation approaches. IEEE Trans. Power Electron. 2003, 18, 1254-1261.

21. Wen, B.; Boroyevich, D.; Burgos, R.; Mattavelli, P.; Shen, Z. Inverse Nyquist Stability Criterion for Grid-Tied Inverters. IEEE Trans. Power Electron. 2017, 32, 1548-1556. [CrossRef]

22. Liu, F.; Liu, J.; Zhang, H.; Xue, D. Stability Issues of Z + Z Type Cascade System in Hybrid Energy Storage System (HESS). IEEE Trans. Power Electron. 2014, 29, 5846-5859. [CrossRef]

23. Liu, Z.; Liu, J.; Bao, W.; Zhao, Y. Infinity-Norm of Impedance-Based Stability Criterion for Three-Phase AC Distributed Power Systems with Constant Power Loads. IEEE Trans. Power Electron. 2015, 30, 3030-3043. [CrossRef]

24. Amin, M.; Molinas, M.; Lyu, J.; Cai, X. Impact of Power Flow Direction on the Stability of VSC-HVDC Seen from the Impedance Nyquist Plot. IEEE Trans. Power Electron. 2017, 32, 204-8217. [CrossRef]

25. Zhang, X.; Ruan, X.; Tse, C.K. Impedance-Based Local Stability Criterion for DC Distributed Power Systems. IEEE Trans. Circuits Syst. I Reg. Pap. 2015, 62, 916-925. [CrossRef]

26. Rodríguez, A.; Vázquez, A.; Lamar, D.G.; Hernando, M.M.; Sebastián, J. Different Purpose Design Strategies and Techniques to Improve the Performance of a Dual Active Bridge With Phase-Shift Control. IEEE Trans. Power Electron. 2015, 30, 790-804. [CrossRef]

27. Liu, J.; Miura, Y.; Ise, T. Comparison of Dynamic Characteristics between Virtual Synchronous Generator and Droop Control in Inverter-Based Distributed Generators. IEEE Trans. Power Electron. 2016, 31, 3600-3611. [CrossRef]

28. Yin, B.; Oruganti, R.; Panda, S.K.; Bhat, A.K.S. A Simple Single-Input-Single-Output (SISO) Model for a Three-Phase PWM Rectifier. IEEE Trans. Power Electron. 2009, 24, 620-631. [CrossRef]

29. Wang, L.; Zhang, D.; Wang, Y.; Wu, B.; Athab, H.S. Power and Voltage Balance Control of a Novel Three-Phase Solid-State Transformer Using Multilevel Cascaded H-Bridge Inverters for Microgrid Applications. IEEE Trans. Power Electron. 2016, 31, 3289-3301. [CrossRef]

30. Li, Y.; Zhang, H.; Liang, X.; Huang, B. Event-Triggered Based Distributed Cooperative Energy Management for Multi-Energy Systems. IEEE Trans. Ind. Inform. 2018, in press.

31. Cvetkovic, I.; Boroyevich, D.; Mattavelli, P.; Lee, F.C.; Dong, D. Unterminated Small-Signal Behavioral Model of DC-DC Converters. IEEE Trans. Power Electron. 2013, 28, 1870-1879. [CrossRef] 